\title{
Facial enhancement using biomimetic oral appliance therapy in adults
}

\author{
G. Dave Singh ${ }^{1 *}$, Kwang Yeon Kim $^{2}$ and Derek Preble ${ }^{3}$ \\ ${ }^{1}$ BioModeling Solutions, Inc., 17933 NW Evergreen Parkway, Suite 280, Beaverton, OR 97006, USA \\ ${ }^{2}$ Seoul, South Korea, \\ ${ }^{3}$ Derek Preble, Salt Lake City, USA
}

\begin{abstract}
Biomimetic oral appliance therapy (BOAT) has been shown to increase midfacial bone volume in adults non-surgically. This study tests the hypothesis that facial enhancement can also be achieved using BOAT. In this investigation, 12 adults underwent BOAT by a dentist with advanced training in BOAT. The following craniofacial parameters were measured on standardized lateral photographs; frontonasal angle; nasolabial angle; labiomental angle, and thyromandibular angle. The mean configurations were also subjected to statistical analyses, principal components analysis (PCA), and finite-element analysis (FEA). The results showed that the mean labiomental angle improved from $126.3^{\circ}$ to $134.0^{\circ}(\mathrm{p}<0.01)$ and the mean thyromandibular angle improved from $126.5^{\circ}$ to $118.6^{\circ}$ ( $\mathrm{p}<0.01$ ). The mean, preand post-treatment craniofacial configurations, however, were not statistically different when tested using PCA (p>0.05), with the first two principal components accounting for approx. $70 \%$ of the total shape change. But, using FEA, the submandibular region showed a relative $32 \%$ decrease in size and the labiomental region also showed a $20 \%$ relative size-decrease. Therefore, this study supports the notion that BOAT may enhance facial appearance non-surgically in adults.
\end{abstract}

\section{Introduction}

Currently, much emphasis is being placed on understanding the process of human facial aging, and patients often request enhancement of their facial esthetics from dental personnel. For example, since loss of muscle tone and supportive fat promotes wrinkling and sagging of the skin, dermal filling agents for soft tissue augmentation have been used in the past [1]. But, individuals age at different rates, and these changes become apparent as wrinkles, accentuation of facial lines, laxity and dependency of the facial skin [2]. Thus, repeated applications of moisturizing creams, oils, and cosmetics may be helpful in hydrating the facial skin and improving its appearance, while chemosurgery (peeling) and dermabrasion may also reduce facial lines and wrinkles [3]. In addition, collagen implants may improve facial contours. However, the collagen later migrates into subcutaneous planes, which explains the loss of correction [4]. Alternatively, Botox (botulinum neurotoxin) may be used, but despite its ubiquity in cosmetic circles and broad general awareness, there is a dearth of studies on botulinum regarding facial cosmesis [5].

On the other hand, the non-Caucasian face has various unique attributes, including skin tone and subcutaneous fat content, and these differences may place a patient at increased risk of post-operative scarring and pigmentation. Therefore, it is important to discuss both surgical and non-surgical treatment options for rejuvenation of the non-Caucasian midface, including the periorbital region to meet a patient's expectations [6]. For example, Korean women prefer an ideal eyebrow of middle height with an arched shape [7]. In fact, from an Asian esthetic point of view, a soft facial appearance seems to be more attractive, especially in the gonial angle and mental region from the lateral view to obtain a more slender, oval face. Therefore, Li et al. [8] undertook a combination of surgical osteotomies to reshape the face to make it look more harmonious, particularly in the middle and lower one-third of the face. But, Asians increasingly seek non-surgical facial esthetic treatments, especially at younger ages. In addition, Asians differ from Caucasians in terms of attitudes to beauty, structural facial anatomy, as well as signs and rates of aging. Presumably, these treatment requests to improve facial shape reflect a desire to correct underlying facial structural deficiencies that detract from cultural ideals of facial beauty [9]. For example, Chinese women, who have a characteristic flattened facial contour prefer enhanced projection of the nose, including the nasal dorsum, nasal tip and columella [10].

As noted above, an improved understanding of the anatomy and physiology of the face provides a foundation for a more comprehensive approach to facial rejuvenation, using a holistic approach that considers the entire facial structural framework to provide more natural outcomes [11]. But, the associated phenomena that take place in the underlying bone of the facial skeleton are often overlooked. Skeletally, the maxilla changes its form due to remodeling in a downward direction over time. These bony changes result in a thinner upper lip and result in more tooth-show during smiling, due to an increase in vertical maxillary dimension [12]. The reduced maxillofacial bone volume results also in the deepening of the nasolabial grooves. Thus, retrusion of the facial skeleton below the soft tissue of the nasolabial fold causes the nasolabial fold to appear more prominent in both males and females [12]. Until more recently, some dental professionals have concerned themselves largely with facial development in children, while the esthetic concerns of adults have been somewhat neglected. Recently, however, an increased midfacial bone volume in adults

Correspondence to: Prof. G. Dave Singh, DDSc, PhD DMD, BioModeling Solutions, Inc. 17933 NW Evergreen Parkway, Suite 280 Beaverton, OR 97006, USA, E-mail: info@biomodelings.com

Key words: non -surgical, facial-enhancement, palatal-expansion, adult

Received: July 01, 2016; Accepted: August 01, 2016; Published: August 04, 2016 
treated with a non-surgical, biomimetic oral device was demonstrated [13]. Biomimetic oral appliance therapy (BOAT) was also shown to have beneficial effects in adults with mild, moderate and even severe obstructive sleep apnea [14,15]. But, in addition, this non-surgical, pain-free technique, which does not use drugs or injections, may have other effects, such as improving facial appearance. Therefore, the aim of this study is to test the hypothesis that facial enhancement can be achieved using BOAT in Korean adults.

\section{Materials, methods and sample}

After obtaining informed consent, 12 consecutive patients were recruited for this study ( 9 females; 3 males; mean age $=29$ yrs \pm 3 ). The rights of the subjects were protected by following the Declaration of Helsinki. Inclusion criteria were: adults over age 16yrs; good compliance; no history of hospitalization for craniofacial trauma or surgery; no congenital, craniofacial anomalies, and a fully-dentate upper arch. The exclusion criteria included: age $<16$ yrs; lack of compliance; active periodontal disease; tooth loss during treatment; poor oral hygiene, and systemic bisphosphonate therapy. After careful history-taking and craniofacial examination, standardized, pretreatment lateral photographs were taken for subjects that met these criteria.

Following diagnostics, an upper, biomimetic oral appliance (DNA appliance $\left.{ }^{\circledR}\right)$ was constructed specifically for each individual. This biomimetic device has 6 (patented) 3D axial springs and a labial bow (Figure 1). It includes a midline expansion screw that is advanced approx. $0.25 \mathrm{~mm}$ per week; a width, which is consistent with sutural homeostasis [16]. All subjects who underwent biomimetic oral appliance therapy (BOAT) were treated by a general dentist (KYK) with advanced training in this technique. All subjects were instructed to wear the appliance 12 to16hrs per day, starting in the late afternoon and throughout the night. The appliance was not worn during the day, and was removed for eating and cleaning.

All subjects reported for review each month. At each monthly follow-up, examination for the progress of midfacial development was recorded. Adjustments to the devices were performed to optimize their efficacy. The fitting surface acrylic/posterior border was adjusted gradually to permit palate to remodel inferiorly. The device was selectively equilibrated and the occlusal acrylic reduced to permit the lower teeth to track the occlusion. Onlygentle pressures were transmitted to the teeth, and the functionality of the device was checked with the subject activating a mild force on biting. The subjects were encouraged to maintain their treatment regimen as outlined. Development of the lower arch was implemented using a lower biomimetic appliance to permit arch re-coordination. A lower appliance was implemented after 1-3 months, depending on the patient's progress. On completion of

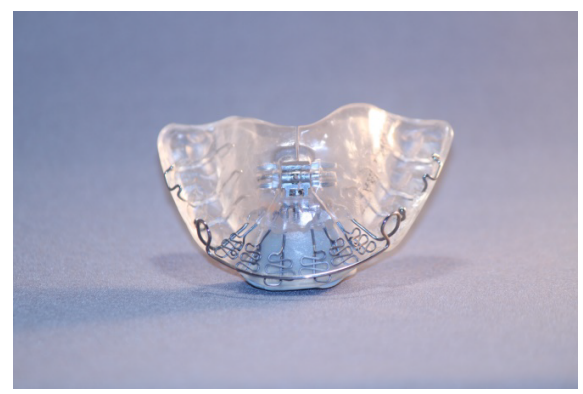

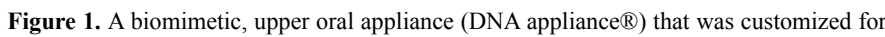
each individual. This biomimetic device has 6 (patented) 3D axial springs and a labial bow. treatment, standardized, post-treatment lateral photographs were taken for all subjects.

Using appropriate software, 12 homologous landmarks (Table 1; Figure 2) were digitized on the pre- and post-treatment lateral photographs. The following mean craniofacial parameters were measured: frontonasal angle; nasolabial angle; labiomental angle, and thyromandibular angle. The measurement parameters are summarized in Figure 2. The findings were subjected to statistical analysis, using paired t-tests. In addition, the mean, pre- and posttreatment craniofacial configurations were computed using Procrustes superimposition. These configurations were subject to principal components analysis (PCA) and finite-element analysis (FEA).

Table 1. Definitions of homologous landmarks digitized on the pre- and post-treatment lateral photographs.

\begin{tabular}{|c|c|c|}
\hline Landmark & Name & Definition \\
\hline 1 & Submandibulare & $\begin{array}{l}\text { Most concave point of the junction of the mandible } \\
\text { with the neck }\end{array}$ \\
\hline 2 & Soft pogonion & Most prominent point of the chin \\
\hline 3 & Soft B point & Most concave point of the labiomental groove \\
\hline 4 & Prolabiale inferior & Most prominent point of the lower lip \\
\hline 5 & Oral commissure & $\begin{array}{l}\text { Junction of the upper and lower lips at the corner of } \\
\text { the mouth }\end{array}$ \\
\hline 6 & Prolabiale superior & Most prominent point of the upper lip \\
\hline 7 & Base of columella & $\begin{array}{l}\text { Most concave point of the junction of the columella } \\
\text { and the upper lip }\end{array}$ \\
\hline 8 & Pronasale & Most prominent point on the tip of the nose \\
\hline 9 & Soft tissue nasion & $\begin{array}{l}\text { Most concave point of the frontonasal suture on the } \\
\text { bridge of the nose }\end{array}$ \\
\hline 10 & Soft tissue glabella & $\begin{array}{l}\text { Most prominent point of the frontal bone on the } \\
\text { forehead }\end{array}$ \\
\hline 11 & $\begin{array}{l}\text { Laryngeal } \\
\text { prominence }\end{array}$ & $\begin{array}{l}\text { Most prominent point of the thyroid cartilage in the } \\
\text { neck }\end{array}$ \\
\hline
\end{tabular}

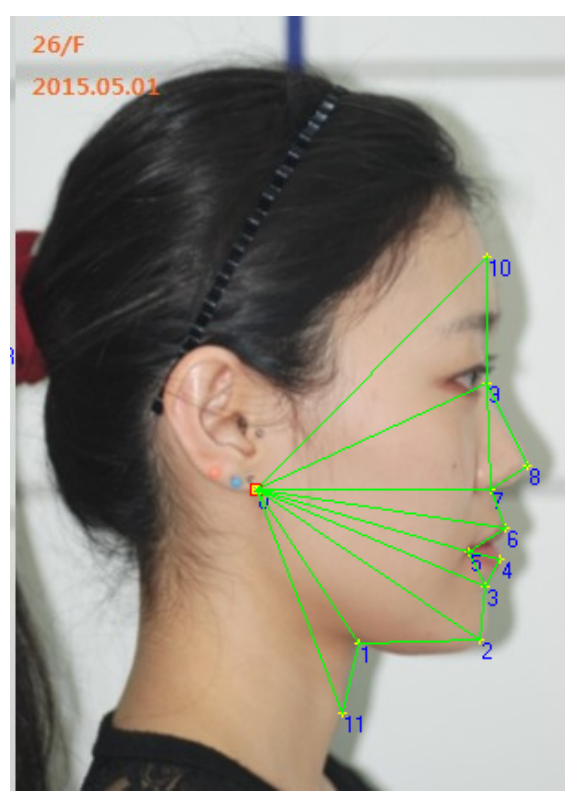

Figure 2. The craniofacial parameters that were measured in this study were: Frontonasal angle: Angle between 10-9-8 Nasolabial angle: Angle between 8-7-6

Labiomental angle: Angle between 4-3-2

Thyromandibular angle: Angle between 2-1-11. 


\section{Results}

The 12 adults that participated in this study had a mean age of 29 yrs \pm 8 . The mean treatment time was 11.2 mos. \pm 7 . Figure 3 shows the pre- and post-treatment facial features. Overall, the midface appears fuller with less pronounced labiomental and thyromandibular angles. The mean changes in the craniofacial parameters measured are summarized in Table 2. Specifically, the mean labiomental angle improved from $126.3^{\circ}$ \pm 11.3 to $134.0^{\circ} \pm 7.3(\mathrm{p}<0.01)$, and the mean thyromandibular angle improved from $126.6^{\circ} \pm 11.2$ to $118.6^{\circ} \pm 14.3(\mathrm{p}<0.05)$.

The mean, pre- and post-treatment craniofacial configurations were not statistically different when tested using PCA ( $p>0.05)$, with the first two principal components accounting for approx. $70 \%$ of the total shape change (Figure 4). Using FEA, the submandibular region showed a relative $32 \%$ decrease in size (blue coloration, Figure 5). The direction of change was mostly horizontal (green coloration, Figure 6). The labiomental region also showed a $20 \%$ relative size-decrease (blue coloration, Figure 5).

\section{Discussion}

The popularity of cosmetic procedures for rejuvenating the face has undergone enormous growth over the past few years. However, pain and potential adverse events are challenges for patients undergoing surgical facelifts and other rejuvenating procedures. For example, patients being treated with calcium hydroxyapatite experienced increased pain, erythema, swelling, bruising and redness in the needletreated side compared to using a blunt cannula [17]. Therefore, the current trend is away from surgical interventions and toward noninvasive cosmetic procedures. Non-invasive procedures for achieving a "non-surgical face-lift" include radiofrequency (RF) and infra-red light devices, which stimulate collagen production, as well as electrostimulation of facial muscles. Taub [18] evaluated eye-lift and neck-lift achieved at 12 months, and concluded that an electrical, facial musclestimulating procedure yields significant improvements of the face and neck. In another study, RF in non-ablative skin tightening for skin

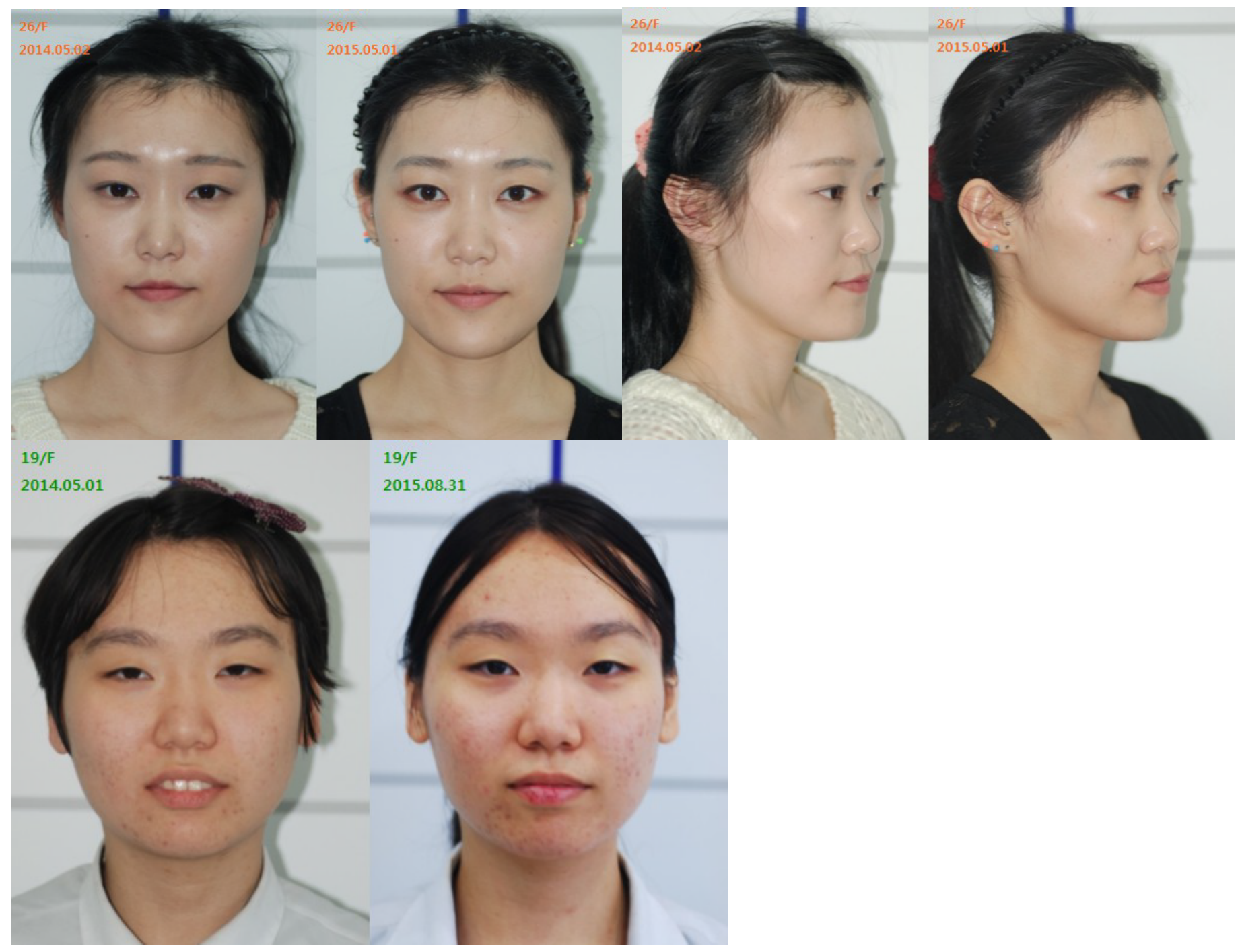

Figure 3. The pre- and post-treatment facial features of subjects that participated in this study. Note that the midface appears fuller with less pronounced labiomental and thyromandibular angles. 
Table 2. The findings of craniofacial parameters measured in this study.

\begin{tabular}{|c|c|c|c|c|c|c|c|c|}
\hline \multirow[b]{2}{*}{ Subject } & \multicolumn{2}{|c|}{ Frontonasal angle } & \multicolumn{2}{|c|}{ Nasolabial angle } & \multicolumn{2}{|c|}{ Labiomental angle } & \multicolumn{2}{|c|}{ Thyromandibular angle } \\
\hline & Pre Tx & Post Tx & Pre $T x$ & Post Tx & Pre Tx & Post Tx & Pre $T x$ & Post Tx \\
\hline 1 & 145.8 & 149.5 & 137.4 & 105.1 & 126.5 & 134.2 & 130 & 124.5 \\
\hline 2 & 135.4 & 139.7 & 87.4 & 81.3 & 138.5 & 128.5 & 110.5 & 95.6 \\
\hline 3 & 138.1 & 139.1 & 82.7 & 90.5 & 118.5 & 128 & 147.4 & 131.4 \\
\hline 4 & 134.9 & 137.3 & 88.2 & 86.8 & 131.8 & 139 & 125.7 & 106.5 \\
\hline 5 & 131.2 & 127.7 & 95.2 & 86.5 & 130.2 & 135.8 & 115.8 & 117.7 \\
\hline 6 & 122 & 116.2 & 101.2 & 101.7 & 150.9 & 147.4 & 110.5 & 106.3 \\
\hline 7 & 138.6 & 144.8 & 71.1 & 78.8 & 119 & 132.9 & 132 & 109.2 \\
\hline 8 & 143.2 & 141.6 & 90.1 & 83.9 & 131.7 & 145.9 & 134.7 & 148 \\
\hline 9 & 138 & 137.9 & 78.9 & 81.8 & 114.7 & 132.7 & 122.2 & 115.4 \\
\hline 10 & 139.8 & 135.4 & 89.6 & 88.7 & 113.3 & 121.5 & 140.6 & 133.5 \\
\hline 11 & 142.4 & 143.7 & 76 & 81.4 & 127.7 & 132.7 & 123.8 & 122.4 \\
\hline 12 & 135.2 & 137.4 & 68.1 & 66.8 & 113.1 & 129.4 & 125.7 & 112.8 \\
\hline Mean & 137 & 137.5 & 86.8 & 86.1 & 126.3 & 134 & 126.6 & 118.6 \\
\hline Std & 5.9 & 8.2 & 12.6 & 10.1 & 11.3 & 7.3 & 11.2 & 14.3 \\
\hline P Value & NS & & NS & & 0.004 & & 0.009 & \\
\hline
\end{tabular}

- Pre

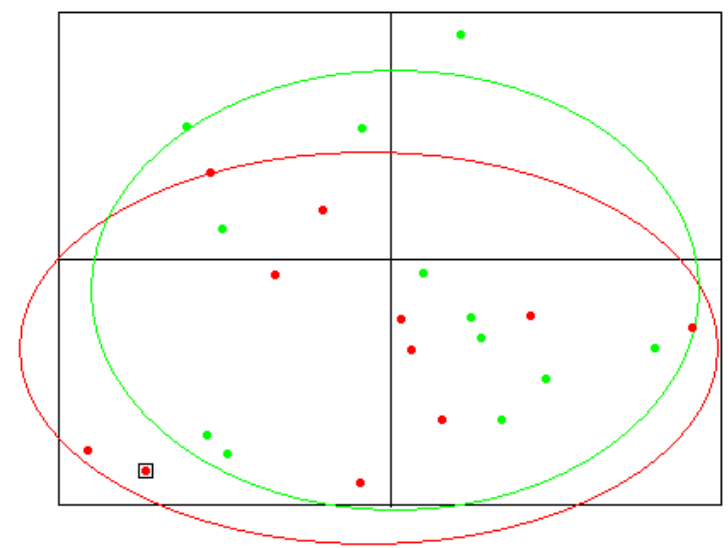

Figure 4. Principal components analysis, showing that the mean, pre- and post-treatment craniofacial configurations were not statistically different when tested using PCA ( $p>$ 0.05 ), with the first two principal components accounting for approx. $70 \%$ of the total shape change.

laxity was assessed in Asians. The investigators judged treatment of nasolabial folds, marionette lines, and sagging jowls. The RF treatment was found to be effective at the 6-month evaluation [19]. In this regard, the mean treatment time this present study was approx. 11 months, thus it is important for the clinician to help their patient understand and select the most appropriate rejuvenating treatment based on a variety of factors, such as timing, age, ethnicity, motivating factors, area to be addressed, and desired outcome [20].

Restoration of facial volume by surgically-redistributing the facial fat compartments may assuage the effects of ageing. But, research continues to suggest the importance of bony remodeling in facial aging [21]. The results of this particular study highlight the importance of skeletal remodeling in improving the soft-tissue contours of the face (Figure 3). Facial wrinkles, however, are not a single groove, but comprise a functional unit, which participates in facial functions responsible for expression, protection, and communication. Thus,

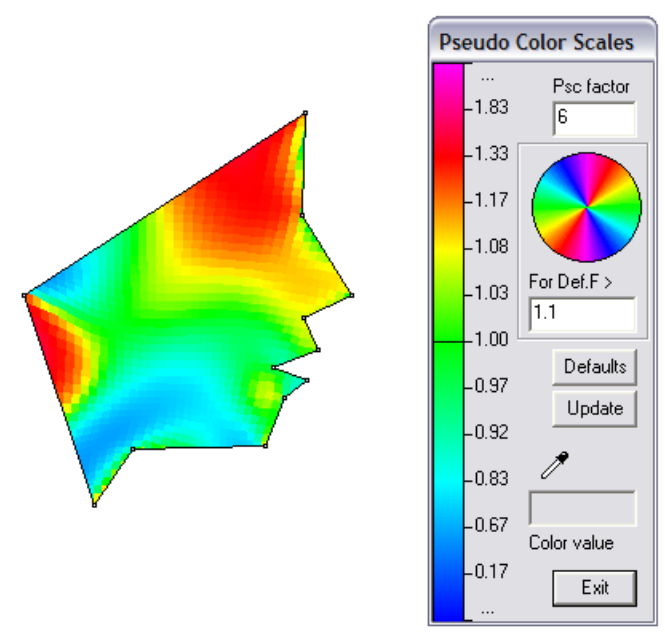

Figure 5. Using FEA, the submandibular region shows a relative $32 \%$ decrease in size (blue coloration). The labiomental region also shows a $20 \%$ relative decrease in size (blue coloration).

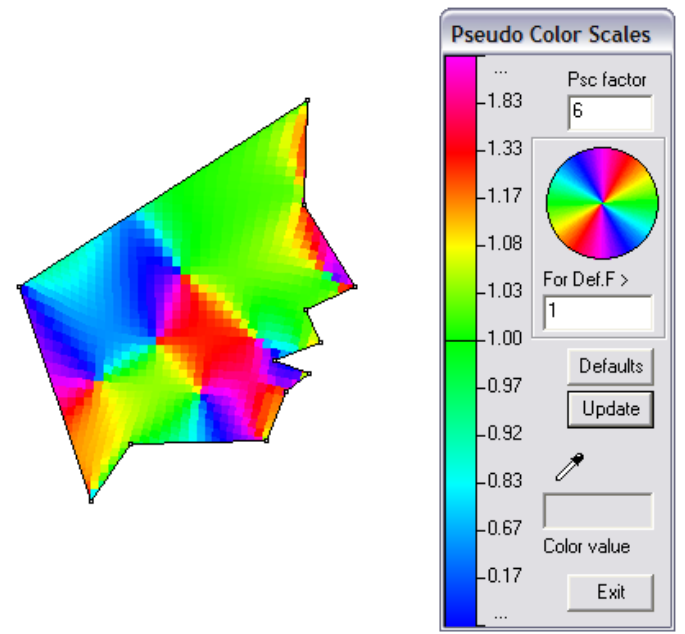

Figure 6. The direction of the facial change was mostly forwards in the horizontal axis, using the circular, pseudocolor scale (green coloration). 
while wrinkles are related to increased muscle tone or contraction [22], the effect of BOAT appears to diminish the appearance of some facial contours, such as the labiomental groove, while enhancing others such as the thyromandibular angle.

Owsley [23] reported on the surgical correction of prominent nasolabial folds by undermining the malar fat pad and fixation by sutures. In contrast, the findings of this particular study suggest that improvements in the nasolabial folds can be also achieved nonsurgically (Figure 3 ). The biomimetic oral device that was worn to promote an increase in midfacial bone volume also achieved overall facial enhancement (Figure 3). The biomimetic device widened the smile by remodeling the maxilla and likely increased maxillary volume [13]. Aging also affects the skin, the facial contour, the dentition and periodontium, the facial and masticatory muscles, the facial skeleton and the temporomandibular joints. Using this novel biomimetic device, however, dental professionals may be able to assist cosmetic/ esthetic facial plastic surgeons and other cosmetology professionals by providing patients with a biomimetic appliance to wear overnight that aims to increase midfacial volume, and remodel the maxilla upward and outwards to prevent and retard the premature aging of the face. Indeed, the mean nasolabial angle improved from $86.7^{\circ} \pm 12.6$ to $86.1^{\circ}$ \pm 10.1 in our study but marginally failed to reach statistical significance $(\mathrm{p}=0.07)$.

In addition to addressing the aging changes of the forehead, nose, eyes and cheeks, the natural progression of these endeavors are to understand aging of the lower one-third of the face and aging of the neck (24). In this present study, we found the mean labiomental angle improved from $126.3^{\circ} \pm 11.3$ to $134.0^{\circ} \pm 7.3(\mathrm{p}<0.01)$. A relatively acute labiomental angle indicates not only a deep bite (which may be associated with temporo-mandibular joint issues) but also a retruded mandible. But mandibular retrognathia is associated with an increased risk of OSA (25). In addition, in our study the thyromandibular angle decreased from $126.6^{\circ} \pm 11.2$ to $118.6^{\circ} \pm 14.3(\mathrm{p}<0.01)$. Lam et al. [26] showed that an increased thyromandibular angle is associated with an increased severity of OSA in both Asian and Caucasian subjects. Thus, we suggest that BOAT might not only be used for facial enhancement but also for the possible prevention of undiagnosed OSA. Therefore, further studies are planned to correlate 3D facial changes with the outcomes of overnight sleep studies to determine whether non-surgical facial enhancement can be used to prevent OSA in both children and adults.

\section{References}

1. Dastoor SF, Misch CE, Wang HL (2007) Dermal fillers for facial soft tissue augmentation. J Oral Implantol 33: 191-204. [Crossref]

2. Fedok FG (1996) The aging face. Facial Plast Surg 12: 107-115. [Crossref]

3. Lubowe II (1976) Treatment of the aging skin by dermatologic methods. J Am Geriatr Soc 24: 25-28. [Crossref]

4. Stegman SJ, Chu S, Bensch K, Armstrong R (1987) A light and electron microscopic evaluation of Zyderm collagen and Zyplast implants in aging human facial skin. A pilot study. Arch Dermatol 123: 1644-1649.
5. Berry MG, Stanek JJ (2012) Botulinum neurotoxin A: a review. J Plast Reconstr Aesthet Surg 65: 1283-1291. [Crossref]

6. Batniji RK1, Perkins SW (2010) Upper and midfacial rejuvenation in the nonCaucasian face. Facial Plast Surg Clin North Am 18: 19-33. [Crossref]

7. Hwang SJ, Kim H, Hwang K, Kim SK, Kim YS, et al. (2015) Ideal or Young-Looking Brow Height and Arch Shape Preferred by Koreans. J Craniofac Surg 26: e412-416. [Crossref]

8. Li X, Hsu Y, Hu J, Khadka A, Chen T, et al. (2013) Comprehensive consideration and design for treatment of square face. J Oral Maxillofac Surg 71: 1761. [Crossref]

9. Liew S, Wu WT, Chan HH, Ho WW, Kim HJ, et al. (2016) Consensus on Changing Trends, Attitudes, and Concepts of Asian Beauty. Aesthetic Plast Surg 40: 193-201. [Crossref]

10. Han X, Hu J, Cheng L, Li F (2015)Multiplane hyaluronic acid (EME) in female Chinese rhinoplasty using blunt and sharp needle technique. J Plast Reconstr Aesthet Surg 68:1504-1509.

11. Sadick NS, Manhas-Bhutani S, Krueger N (2013) A novel approach to structural facial volume replacement. Aesthetic Plast Surg 37: 266-276.[Crossref]

12. Pessa JE, Zadoo VP, Mutimer KL, Haffner C, Yuan C, et al. (1998) Relative maxillary retrusion as a natural consequence of aging: combining skeletal and soft-tissue changes into an integrated model of midfacial aging. Plast Reconstr Surg 102: 205-212.

13. Singh GD, Heit T, Preble D (2014) Changes in 3D midfacial parameters after biomimetic oral appliance therapy in adults. J Ind Orthod Soc 48: 104-108.

14. Singh GD, Griffin TM, Chandrashekhar R (2014) Biomimetic oral appliance therapy in adults with mild to moderate obstructive sleep apnea. Aust J Sleep Dis1: 1-5.

15. Singh GD, Griffin TM, Cress SE (2016) Biomimetic oral appliance therapy in adults with severe obstructive sleep apnea. J Sleep Disord Ther 5: 1-5.

16. Borke JL, Yu JC, Isales CM, Wagle N, Do NN, et al. (2003) Tension-induced reduction in connexin 43 expression in cranial sutures is linked to transcriptional regulation by TBX2. Ann Plast Surg 51: 499-504.[Crossref]

17. Beer KR (2014) Safety and effectiveness of injection of calcium hydroxylapatite via blunt cannula compared to injection by needle for correction of nasolabial folds. $J$ Cosmet Dermato l13: 288-296.[Crossref]

18. Taub AF (2006) Evaluation of a nonsurgical, muscle-stimulating system to elevate soft tissues of the face and neck. J Drugs Dermatol 5: 446-450.[Crossref]

19. Kushikata N1, Negishi K, Tezuka Y, Takeuchi K, Wakamatsu S (2005) Non-ablative skin tightening with radiofrequency in Asian skin. Lasers Surg Med 36: 92-97. [Crossref]

20. Mandy SH (2009) Satisfying patient expectations with soft-tissue augmentation Dermatol Online J 15: 1.[Crossref]

21. Pessa JE, Zadoo VP, Yuan C, Ayedelotte JD, Cuellar FJ, et al. (1999) Concertina effect and facial aging: nonlinear aspects of youthfulness and skeletal remodeling, and why, perhaps, infants have jowls. Plast Reconstr Surg 103: 635-644.[Crossref]

22. Hatzis J (2004) The wrinkle and its measurement--a skin surface Profilometric method. Micron 35: 201-219.[Crossref]

23. Owsley JQ1 (1993) Lifting the malar fat pad for correction of prominent nasolabial folds. Plast Reconstr Surg 91: 463-474.[Crossref]

24. Patel BC (2006) Aesthetic surgery of the aging neck: options and techniques. Orbit 25 327-356.[Crossref]

25. Dahlqvist J1, Dahlqvist A, Marklund M, Berggren D, Stenlund H, et al. (2007) Physical findings in the upper airways related to obstructive sleep apnea in men and women. Acta Otolaryngol 127: 623-630.[Crossref]

26. Lam B, Ip MS, Tench E, Ryan CF (2005) Craniofacial profile in Asian and white subjects with obstructive sleep apnoea. Thorax 60: 504-510.[Crossref]

Copyright: (C2016 Dave Singh G. This is an open-access article distributed under the terms of the Creative Commons Attribution License, which permits unrestricted use, distribution, and reproduction in any medium, provided the original author and source are credited. 